\title{
Commentary: Enhanced recovery after cardiac surgery: A game changer, passing fad, or somewhere in between?
}

\author{
Subhasis Chatterjee, MD, FACS, FACC, FCCP
}

\footnotetext{
From the Michael E. DeBakey Department of Surgery, Divisions of General and Cardiothoracic Surgery, Baylor College of Medicine, Houston; and Division of Cardiovascular Surgery, Texas Heart Institute, Houston, Tex. Disclosures: Author has nothing to disclose with regard to commercial support.

Received for publication Nov 13, 2018; accepted for publication Nov 14, 2018; available ahead of print Jan 8, 2019.

Address for reprints: Subhasis Chatterjee, MD, FACS, FACC, FCCP, Baylor College of Medicine, One Baylor Plaza, MS: BCM 390, Houston, TX 77030-3411 (E-mail: subhasis.chatterjee@ bcm.edu).

J Thorac Cardiovasc Surg 2019;157:1889-90

$0022-5223 / \$ 36.00$

Copyright (c) 2018 by The American Association for Thoracic Surgery

https://doi.org/10.1016/j.jtcvs.2018.11.046
}

In this issue of The Journal, Williams and colleagues ${ }^{1}$ describe the largest published and first North American study on the feasibility and efficacy of an enhanced recovery after surgery (ERAS) program in cardiac surgery. In the 1990s, ERAS was first established in Europe for major abdominal surgery and has since been extended across several surgical procedures and specialties. ${ }^{2}$ A group in the United Kingdom ${ }^{3}$ (105 patients) and another in France ${ }^{4}$ (71 patients) have previously reported their ERAS experience in cardiac surgery, showing shorter intensive care unit (ICU) and hospital length of stay (LOS).

This 932-patient study by Williams and colleagues ${ }^{1}$ showed comparable results, demonstrating an institutional commitment to evidence-based ERAS recommendations for clinical pathways to optimize preoperative, intraoperative, and postoperative care. Although concepts such as earlier extubation and mobility are widely accepted, others such as carbohydrate loading before surgery appear less intuitive. The barriers to implementing an ERAS program in cardiac surgery stem from justifiable concerns that these patients are vulnerable from the effects of cardiopulmonary bypass, hypothermia, and other physiologic derangements; as a result, real-world experiences are instructive.

But is ERAS new or just "fast-track" by another name? Fast-track and rapid recovery have been part of cardiac surgery for more than 2 decades. Although the focus was initially reducing intraoperative analgesia to reduce the time to ventilator liberation, other fast-track programs have comprehensively included preoperative education, early extubation, prevention of postoperative nausea and vomiting, and earlier rehabilitation and discharge, ${ }^{5}$ similar to the current ERAS strategies. Perhaps the renewed impetus now is that, as the expected mortality rate for patients who undergo coronary bypass, aortic valve replacement, or mitral valve repair (composing 80\% of heart surgeries) approaches $1 \%$ to $1.5 \%$, a program will be gauged by "softer" quality metrics (eg, LOS). Moreover,

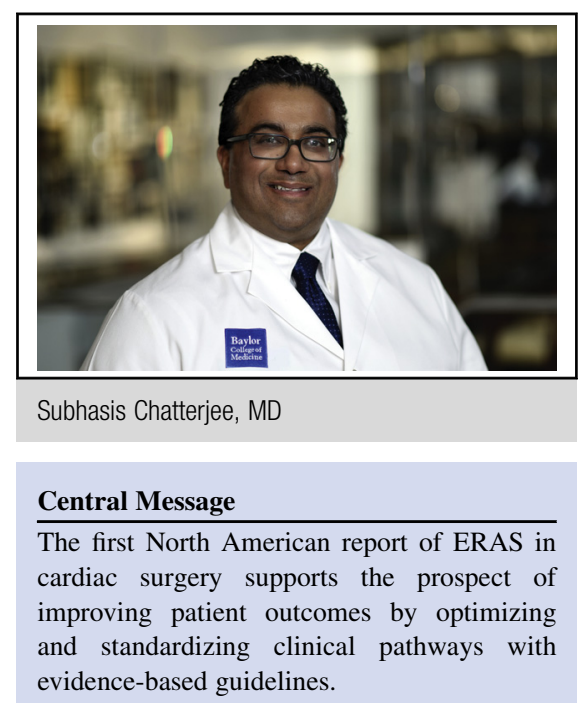

See Article page 1881 . this will be emphasized even more as minimally invasive and transcatheter options in "low-risk" patients increasingly become part of the landscape. Although much of critical care is justifiably focused on organ dysfunction and device management for the "sickest of the sick," process efficiency and enhancing value are central components of critical care in low-risk patients undergoing low-risk operations.

In their study, Williams and colleagues ${ }^{1}$ demonstrated a substantial 15-hour reduction in ICU LOS after the implementation of ERAS. This was despite having a mixed cohort of coronary artery bypass grafting and valve procedure patients with a percentage of intra-aortic balloon pump use $(16 \%-17 \%)$ twice as high as the Society of Thoracic Surgeons database average ${ }^{6}$ and a prevalence of previous stroke $(21 \%-23 \%) 3$ times higher than the Society of Thoracic Surgeons database average. ${ }^{6}$ Because the median extubation time was unchanged ( 5 hours), it raises the possibility of whether the observed reduction in ICU LOS was influenced by the earlier removal of intra-aortic balloon pumps, the more rapid weaning of vasoactive medications, or the earlier availability of step-down beds. However, if we apply the British cycling coach Sir David Brailsford's concept of "aggregation of marginal gains" to surgical care, small incremental gains can cumulatively result in a benefit and improved outcomes. 
This may explain the observed reduction in ICU LOS, whereby small amounts of progress across several fronts may have collectively resulted in a benefit. It is most likely true that not all marginal gains are equally sized. Identifying those measures that provide more benefit may streamline the ERAS process and facilitate its adoption by skeptics. Indeed, many programs already perform several of these individual processes without being formally operationalized as an "ERAS protocol." It is reassuring that implementation of ERAS and a shorter LOS did not come at the expense of increased readmissions or deviations in glycemic control.

In addition, the current opioid epidemic makes counteractive efforts such as ERAS' multimodal analgesia strategy both timely and necessary. The prolonged use of opioids after 90 days has been observed in 3\% of patients after coronary artery bypass grafting and in $6 \%$ to $9 \%$ after thoracic surgery. ${ }^{7}$ Although Williams and colleagues ${ }^{1}$ examined opiate use during the first 24 hours after surgery, determining whether opioid use was reduced during the entire hospitalization or the first 30 to 90 days after surgery would more definitively demonstrate success.

In conclusion, one may ask how much of an effect occurred because of the Hawthorne effect, ${ }^{8}$ whereby people perform better because they are being observed. Perhaps the key to ERAS is the sustained commitment to process improvement and knowing that those results are being tracked and, ultimately, judged.

Editorial support was provided by Scientific Publications at the Texas Heart Institute.

\section{References}

1. Williams JB, McConnell G, Allender JE, Woltz P, Kane K, Smith PK, et al. Oneyear results from the first US-based enhanced recovery after cardiac surgery (ERAS Cardiac) program. J Thorac Cardiovasc Surg. 2019;157:1881-8.

2. Ljungqvist $\mathrm{O}$, Scott M, Fearon KC. Enhanced recovery after surgery: a review. JAMA Surg. 2017;152:292-8.

3. Fleming IO, Garratt C, Guha R, Desai J, Chaubey S, Wang Y, et al. Aggregation of marginal gains in cardiac surgery: feasibility of a perioperative care bundle for enhanced recovery in cardiac surgical patients. J Cardiothorac Vasc Anesth. 2016;30:665-70.

4. Zaouter C, Imbault J, Labrousse L, Abdelmoumen Y, Coiffic A, Colonna G, et al. Association of robotic totally endoscopic coronary artery bypass graft surgery associated with a preliminary cardiac enhanced recovery after surgery program: a retrospective analysis. J Cardiothorac Vasc Anesth. 2015;29:1489-97.

5. Engelman RM, Rousou JA, Flack JE III, Deaton DW, Humphrey CB, Ellison LH, et al. Fast-track recovery of the coronary bypass patient. Ann Thorac Surg. 1994; 58:1742-6.

6. ElBardissi AW, Aranki SF, Sheng S, O’Brien SM, Greenberg CC, Gammie JS Trends in isolated coronary artery bypass grafting: an analysis of the Society of Thoracic Surgeons adult cardiac surgery database. J Thorac Cardiovasc Surg. 2012;143:273-81.

7. Clarke H, Soneji N, Ko DT, Yun L, Wijeysundera DN. Rates and risk factors for prolonged opioid use after major surgery: population based cohort study. BMJ. 2014;348:g1251.

8. McCambridge J, Witton J, Elbourne DR. Systematic review of the Hawthorne effect: new concepts are needed to study research participation effects. J Clin Epidemiol. 2014;67:267-77. 